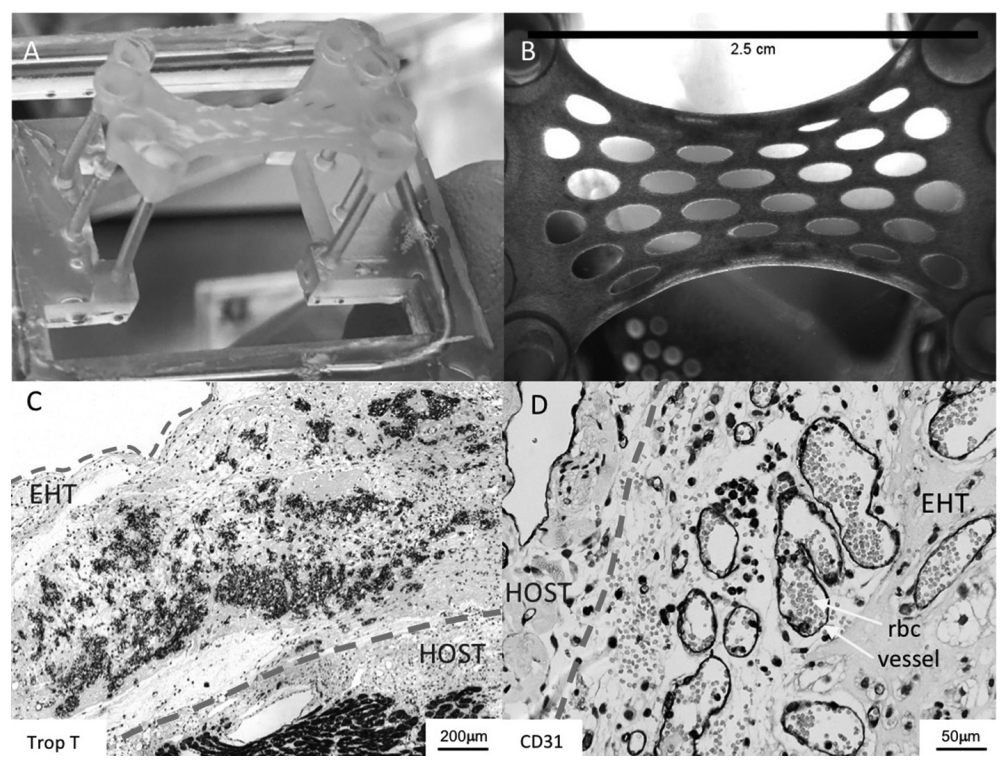

Abstract BS27 Figure 1 A/B) EHT Images; C) 20x troponin T(brown) of rabbit myocardium/EHT(2 weeks after grafting), blue counterstain=haematoxylin, red lines=EHT borders; D) 63x CD31 staining(brown) rabbit/EHT border zone(2 weeks after grafting), blue stain=haematoxylin, red lines=graft/host border zones

Conclusion We successfully upscaled hiPSC-CM derived EHT to a clinically relevant size and were able to demonstrate feasibility and integration using a rabbit model of myocardial infarction. Tissue engineering strategies may be the preferred modality of cell delivery for future cardiac regenerative medicine studies.

Conflict of interest none

\section{BS28 ENDOTHELIAL STAT5A IS ENRICHED AT ATHEROPRONE REGIONS OF THE AORTA AND DRIVES INFLAMMATION IN RESPONSE TO LOW SHEAR STRESS}

Hannah Roddie*, Maria Fragiadaki, Paul Evans. ${ }^{1}$ The University of Sheffield

\subsection{6/heartjnl-2019-BCS.191}

Introduction Atherosclerosis is an inflammatory disease that develops at bends and branches of the arteries that are exposed to disturbed blood flow which generates low wall shear stress (WSS). These haemodynamic conditions lead to altered endothelial function and promote proliferation, apoptosis and inflammatory activation of endothelial cells (ECs), as well as other fundamental processes that drive disease. The Janus Kinase and Signal Transducer and Activators of Transcription (JAK/STAT) is an evolutionarily conserved pathway with key roles in the control of proliferation, apoptosis and inflammatory activation, yet its role in atherosclerosis is poorly understood despite the fact that these processes drive atherogenesis.

Methods Microarrays were performed on ECs from the porcine aorta to quantify stat5a expression in low WSS (atheroprone) and high WSS (atheroprotected) regions, and data were validated by qRT-PCR. En face immunostaining of the murine aortic arch was carried out to quantify Stat5a expression at low and high WSS. In vitro studies were performed using human coronary artery endothelial cells (HCAECs) and human umbilical vein endothelial cells (HUVECs) which were subjected to either retroviral-mediated shRNA (HCAECs) or siRNA (HUVECs) silencing of Stat5a. These cells were then exposed to low $\left(5\right.$ dynes $\left./ \mathrm{cm}^{2}\right)$ or high $\left(10\right.$ dynes $\left./ \mathrm{cm}^{2}\right)$ WSS for 72 hours on the orbital system and inflammatory molecule (ICAM-1, VCAM-1, E-Selectin and MCP-1) expression was studied by qRT-PCR.

Results qRT-PCR analysis of the porcine aorta identified that Stat5a was enriched at low WSS regions compared to high WSS regions $(\mathrm{P}<0.05)$. En face staining of the murine aorta also revealed that endothelial Stat5a expression was enhanced at regions of low WSS compared to high WSS $(\mathrm{P}<0.05)$. Similarly, Stat5a mRNA expression was enhanced in cultured HCAEC exposed to low compared to high WSS. Functional studies showed that silencing of Stat5a led to a reduction of ICAM-1 $(\mathrm{P}<0.05), \quad$ VCAM-1 $\quad(\mathrm{P}<0.05)$, E-Selectin $\quad(\mathrm{P}<0.05)$ and MCP-1 $(\mathrm{P}<0.05)$, indicating that Stat5a is a positive regulator of EC inflammatory activation.

Conclusion Our data demonstrate that Stat5a is upregulated by low WSS both in vivo and in vitro, and that its silencing reduces inflammatory gene expression. These data therefore suggest that Stat5a may regulate the focal nature of atherogenesis by promoting inflammation.

Conflict of interest none

\section{BS29 EXPRESSION OF STABLE SOCS3 IN HUMAN SAPHENOUS VEIN SMOOTH MUSCLE CELLS: POTENTIAL THERAPY FOR VASCULAR RESTENOSIS}

${ }^{1}$ Florah Moshapa*, ${ }^{2}$ Jamie Williams, ${ }^{1}$ Jacobo Ellies, ${ }^{1}$ Kirsten Riches-Suman, ${ }^{3}$ Timothy Palmer. ${ }^{1}$ University of Bradford; ${ }^{2}$ University of Glasgow; ${ }^{3}$ University of Hull

\subsection{6/heartjnl-2019-BCS.192}

Introduction Suppressor of cytokine signalling 3 (SOCS3) limits JAK/STAT pathways involved in vascular inflammation and remodelling responsible for vein graft failure. However, 